〔Med. Entomol. Zool. Vol. 54 No. 3 p. 257-266 2003]

\title{
Laboratory rearing method of Anopheles minimus (Diptera: Culicidae) from Ishigaki Island, the Ryukyu Archipelago, Japan
}

\author{
Yukiko Higa ${ }^{1)}$, Takako Toma ${ }^{1)}$, , Susumu SAita ${ }^{1)}$, \\ Atsuko TAKEI ${ }^{1)}$ and Ichiro MryaGI ${ }^{11}$ \\ 1) Laboratory of Medical Zoology, School of Health Sciences, Faculty of Medicine, \\ University of the Ryukyus, 207 Uehara, Nishihara, Okinawa, 903-0215 Japan \\ 2) Center for Asia-Pacific Island Studies, University of the Ryukyus, \\ 1 Senbaru, Nishihara, Okinawa, 903-0213 Japan
}

(Received: 9 January 2003; Accepted: 2 May 2003)

\begin{abstract}
Biological studies on larval development, insemination and oviposition of Anopheles minimus from Ishigaki Island, the Ryukyu Archipelago, were conducted under laboratory conditions. The larval developmental period was shortened and high pupation and emergence rates were obtained when larvae were reared under the conditions of the non-aeration method, standard food volume and 200-300 larvae per container. Insemination rate was increased with adult density per cage as well as cage size. The number of eggs per fed female was dependent on the method for oviposition. The study showed the following conditions to be suitable for rearing and obtaining a large number of An. minimus originated from Ishigaki Island, the Ryukyus: (1) the drip method for larval rearing should be used in the early generation to estimate suitable food volume and after that, by non-aeration; (2) 200-300 larvae per pan with food volume of $25 \mathrm{mg}$ for the $1 \mathrm{st}, 50 \mathrm{mg}$ for the $2 \mathrm{nd}, 100 \mathrm{mg}$ for the $3 \mathrm{rd}$ and $150 \mathrm{mg}$ for the 4th-instar larvae; (3) adult cage larger than at least the medium-sized cage; and (4) individual oviposition method in small vials.
\end{abstract}

Key words: Anopheles minimus, Ishigaki Island, Ryukyu Archipelago, laboratory rearing

\section{INTRODUCTION}

Anopheles minimus Theobald is widely distributed in rural areas from the Ryukyu Archipelago, Japan, to Southeast Asia and Assam, India. It is an effective vector of rural malaria in these countries. Many field studies have been conducted to examine the insecticide impact on behavior (Nutsathapana et al., 1986a), feeding behavior such as host preference and endo/exo-phagy (Nutsathapana et al., 1986b; Dev, 1996), and temporal and spatial movement of adults (Tsuda et al., 1999a, b). An. minimus in Ishigaki Island, Ryukyu Archipelago was once highly anthrophilic but recently has been suggested as zoophilic also (Miyagi and Toma, 1978).

In addition to field studies, laboratory colonization is important for performing studies that require large numbers of individuals. Wilkinson et al. (1974) and Ye et al. (1982) attempted to colonize An. minimus. They showed larval mortality to be related to rearing water conditions and larval density, and it was difficult for the species to mate in limited space under laboratory conditions, which were critical factors for successful colonization of $A n$. minimus. In spite of repeated attempts, Muirhead-Thomson (1941) failed to colonize this species by natural mating in $60 \times$ $60 \times 60 \mathrm{~cm}$ sized cage. Somboon and Suwonkerd (1997) established a stenogamous colony of An. minimus species A in Chiang 
Mai, Thailand. These studies suggested that successful colonization was dependent on larval rearing and mating under laboratory conditions.

Detailed taxonomical studies and biochemical and molecular techniques have revealed that $A n$. minimus is genetically diverse among populations and constitute a species complex. Important behaviors such as host preference also vary according to each species type in the complex (Kanda et al., 1984; Yuan, 1987; Komalamisra, 1989; Christopher et al., 1990; Sucharit et al., 1988, 1995; Sawabe et al., 1996; Van Bortel et al., 1999). This indicates that information gathered on An. minimus collected in various localities so far may not be wholly applicable to An. minimus of all regions. The Ryukyu Archipelago, Japan, is the northernmost point of $A n$. minimus, and there people suffered from malaria before and after World War II (Miyagi et al., 1996). Somboon et al. (2001) had recently demonstrated that $A n$. minimus in the Ryukyu Archipelago, Japan, could be treated as a new sibling species as evidenced by reproductive isolation and genetic difference from other $A n$. $\mathrm{min}$ imus in Asia. It was important to gather more information such as biology, molecular biology and taxonomy from the aspects of the new sibling species, however, there have been few studies on $A n$. minimus in the Ryukyu Archipelago because of the relative difficulty of colonization.

Toward obtaining biological information for establishment and maintenance of a laboratory colony of $A n$. minimus from Ishigaki Island in which a taxonomical study of the species was formally conducted by Somboon et al. (2001), it is essential to know the effect of at least the rearing conditions on larval development, mating, feeding and oviposition behavior of the species. In the present study, these subjects were examined.

\section{Materials and Methods}

An. minimus used in the present study originated from about 500 larvae collected in May 1994 from Ishigaki Island, located $360 \mathrm{~km}$ south of the main island, Okinawa. All experiments were conducted in an insectarium at $25^{\circ} \mathrm{C}$ and $80 \%$ relative humidity under a 16:8 (L:D) photoperiod. Larvae were reared in plastic pans $(40 \times 30$ $\times 6 \mathrm{~cm})$ containing dechlorinated water. Water volume was kept constant at 3 liters. Larvae were fed a $1: 1$ mixture of mouse pellets and dry yeast twice a day. Standard food volumes were $25 \mathrm{mg}$ for 1st, $50 \mathrm{mg}$ for $2 \mathrm{nd}, 100 \mathrm{mg}$ for 3rd and 150 $\mathrm{mg}$ for 4th-instar larvae per pan, commencing with 200 1st-instar larvae. When $30-50 \%$ of larvae in a pan developed to the next instar, food volume given to larvae was increased according to the regimen. Pupae were removed from the rearing pans by a pipette every day, transferred to a small plastic container and placed inside a cage for adults. Usually, every generation was initiated by 800 larvae from the former generation, and about 300 males and females respectively were produced. Excess of individuals other than the 800 initial larvae were used for experiments. In the first 7 generations, $60 \times 60 \times 30 \mathrm{~cm}$ sized adult cage (large cage) was used for adaptation to laboratory conditions. Ten days after emergence, feeding and insemination rates were examined for females survived in the large cage. For the insemination rate, 15 females in the cage were taken and dissected to check the presence of sperms in spermathecae. After the 8th generation, $20 \times 20$ $\times 30 \mathrm{~cm}$-cage (medium cage) was used for adult experiments. Adults were provided a cotton pad soaked with $3 \%$ sugar solution every 3 days. Anopheles minimus from 6 th to 16 th generation was used for all experiments except for feeding and insemination rates in the large cage.

\section{Larval development}

The effect of rearing methods, larval food volume and larval density on larval development was examined.

Effect of rearing method: Three met- 
hods of larval rearing, "Drip", "Aeration" and "Non-aeration" were used. The drip method was a newly devised method with a siphon system for water circulation (Toma et al., 2001). The water drips from a plastic bottle tap run down from one container to another. The bottom-most container acted as a collecting reservoir (without a tubal outlet) that was emptied every day. The non-aeration method was quite simple, easy to handle and conventional in that the rearing pans were just left undisturbed with no devices. However, scum sometimes formed on the water surface, which might cause bad water quality and, therefore, larval mortality. The aeration method has been used for Aedes and Culex mosquito rearing in many laboratories (Gerberg et al., 1994). It was quite useful to prevent scum formation. Two hundred larvae were reared with each rearing method and fed twice a day according to the food regimen.

Effect of food volume: Two hundred larvae were fed 0.5, 1 (standard) and 1.5 times of standard food volume of the regimen, respectively, and reared by nonaeration method.

Effect of larval density: Using the nonaeration method, larvae were reared with 100,200,300, 400 and 500 individuals per pan. The amount of food per larva was kept constant among different densities. For example, 100 larvae were given half of the standard food volume and 300 larvae were given 1.5 times the standard.

\section{Insemination rate}

Insemination rate was examined under three conditions. Fifteen pairs of newly emerging adults were introduced into a small cage of $16 \times 12 \times 12 \mathrm{~cm}$, and 15 or 30 pairs in medium cages. The effect of adult density was observed by comparison of 15 vs 30 pairs in a medium cage, while the effect of space was provided by the small vs the medium cages containing 15 pairs. Ten small cages with 15 pairs, and 8 medium cages each with 15 pairs and 30 pairs were prepared. One cage each from the three conditions was randomly selected every two days, and all females in the cages were dissected to check for the presence of sperms in spermathecae. The experiment was conducted with individuals from 7 th to 13 th generations when a sufficient number was obtained, and repeated 3-4 times.

For one aspect of the mating behavior, the number of females inseminated by a single male was examined. One male and 20 females that emerged on the same day were released into a medium cage. On the 16 th day after emergence, all females in the cage were dissected to check for insemination. The experiment was repeated 7 times.

\section{Oviposition}

The number of eggs laid was examined, when different oviposition methods were used. Three days after blood feeding, females were introduced singly into small plastic vials $(3.0(\mathrm{D}) \times 5.0(\mathrm{H}) \mathrm{cm})$ with a small amount of water; this was the individual oviposition method. Fifteen females were housed in a small/medium cage for the group oviposition method. A plastic container $(8.0(\mathrm{D}) \times 5.0(\mathrm{H}) \mathrm{cm}) \quad$ containing water was provided for females to oviposit freely in small and medium cages. The total number of eggs oviposited was counted, and the number of eggs per fed female was calculated.

\section{Statistical analyses}

The effects of rearing methods, larval food volume and larval density on the number of days to pupation were analyzed as mean developmental day to pupa by ANOVA. For pair-wise comparisons among different conditions of each experiment, Turky's multiple comparison was conducted. The chi-squared test was used to compare the following parameters under different conditions: (1) pupation rate (the number of pupae/the number of larvae $\times 100$ ); (2) emergence rate for larvae (the number of emerged adults/the number of larvae $\times 100$ ) and pupae (the 
Table 1. Larval develpmental attributes of a sibling species of Anopheles minimus from Ishigaki Island, the Ryukyu Archipelago.

\begin{tabular}{|c|c|c|c|c|c|c|c|c|c|c|}
\hline Experiment & & No. $\exp$ & $\begin{array}{c}\text { Total larvae } \\
\text { used }\end{array}$ & $\begin{array}{l}\text { Days to } \\
\text { pupae }\end{array}$ & Pupation \% & EmergenceL \%1) & EmergenceP \% ${ }^{2)}$ & No. 우 & No. $\sigma^{7}$ & 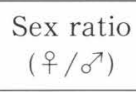 \\
\hline \multirow[t]{5}{*}{ Method } & Drip & 4 & 800 & $13.9 \pm 1.2^{\mathrm{c}}$ & $87.4^{\mathrm{a}}$ & $82.3^{\mathrm{a}}$ & $94.1^{\mathrm{a}}$ & 363 & 295 & $1.23^{\mathrm{a}}$ \\
\hline & Non-aeration & 4 & 800 & $14.4 \pm 1.3^{\mathrm{b}}$ & $83.6^{a}$ & $79.4^{\mathrm{a}}$ & $94.9^{\mathrm{a}}$ & 322 & 313 & $1.03^{\mathrm{a}}$ \\
\hline & Aeration & 2 & 400 & $22.1 \pm 3.4^{\mathrm{a}}$ & $9.5^{\mathrm{b}}$ & $3.3^{\mathrm{b}}$ & $34.2^{\mathrm{b}}$ & 9 & 4 & $2.25^{\mathrm{a}}$ \\
\hline & Statistics & & & $F=678.7$ & $\chi^{2}=887.8$ & $\chi^{2}=851.1$ & $\chi^{2}=203.9$ & & & $\chi^{2}=3.9$ \\
\hline & Prob. & & & $P<0.001$ & $P<0.001$ & $P<0.001$ & $P<0.001$ & & & $P>0.05$ \\
\hline \multirow[t]{5}{*}{ Food } & 0.5 & 4 & 800 & $14.4 \pm 2.0^{\mathrm{a}}$ & $82.1^{\mathrm{b}}$ & $67.6^{\mathrm{b}}$ & $82.3^{\mathrm{a}}$ & 311 & 230 & $1.35^{\mathrm{a}}$ \\
\hline & 1.0 & 4 & 800 & $12.9 \pm 1.3^{\mathrm{b}}$ & $92.5^{\mathrm{a}}$ & $78.0^{\mathrm{a}}$ & $84.3^{\mathrm{a}}$ & 357 & 267 & $1.34^{\mathrm{a}}$ \\
\hline & 1.5 & 2 & 400 & $12.8 \pm 1.3^{\mathrm{b}}$ & $86.0^{\mathrm{b}}$ & $67.3^{\mathrm{b}}$ & $78.2^{\mathrm{b}}$ & 183 & 86 & $2.13^{\mathrm{b}}$ \\
\hline & Statistics & & & $F=188.6$ & $\chi^{2}=38.7$ & $\chi^{2}=26.1$ & $\chi^{2}=6.1$ & & & $\chi^{2}=10.4$ \\
\hline & Prob. & & & $P<0.001$ & $P<0.001$ & $P<0.001$ & $P<0.05$ & & & $P<0.01$ \\
\hline \multirow[t]{7}{*}{ Density } & 100 & 4 & 400 & $12.3 \pm 1.0^{c}$ & $97.8^{\mathrm{a}}$ & $89.0^{\mathrm{a}}$ & $91.0^{\mathrm{ab}}$ & 205 & 151 & $1.36^{\mathrm{a}}$ \\
\hline & 200 & 2 & 400 & $13.0 \pm 1.6^{\mathrm{b}}$ & $94.8^{\mathrm{a}}$ & $87.0^{\mathrm{a}}$ & $91.8^{\mathrm{ab}}$ & 197 & 151 & $1.30^{\mathrm{a}}$ \\
\hline & 300 & 6 & 1,800 & $12.8 \pm 1.5^{\mathrm{b}}$ & $85.1^{\mathrm{b}}$ & $72.8^{\mathrm{b}}$ & $85.6^{c}$ & 710 & 600 & $1.18^{\mathrm{a}}$ \\
\hline & 400 & 3 & 1,200 & $12.5 \pm 1.6^{\mathrm{c}}$ & $60.4^{\mathrm{c}}$ & $56.9^{\mathrm{c}}$ & $94.2^{\mathrm{a}}$ & 308 & 375 & $0.82^{\mathrm{b}}$ \\
\hline & 500 & 4 & 2,000 & $18.5 \pm 2.5^{\mathrm{a}}$ & $34.0^{\mathrm{d}}$ & $30.5^{\mathrm{d}}$ & $89.8^{b}$ & 284 & 326 & $0.87^{\mathrm{b}}$ \\
\hline & Statistics & & & $F=1,518.1$ & $\chi^{2}=1,496.6$ & $\chi^{2}=1,070.0$ & $\chi^{2}=44.4$ & & & $\chi^{2}=29.9$ \\
\hline & Prob. & & & $P<0.001$ & $P<0.001$ & $P<0.001$ & $P<0.05$ & & & $P<0.01$ \\
\hline
\end{tabular}

The values in the same column followed by the same letter were not significantly different $(P>0.05)$ by ANOVA for days to pupae and $\chi^{2}$ test for pupation, emergenceL and emergenceP $\%$.

1) emergence rate to the number of larvae

2) emergence rate to the number of pupae 
number of emerged adults/the number of pupae $\times 100$ ); and (3) sex ratio. If significant difference was detected among the group, multiple comparisons with Bonferroni correction were applied.

\section{RESUlts}

\section{Larval development}

Effect of rearing methods: The mean number of days to pupation was 13.9 days with the drip method, 14.4 days with the non-aeration method, and 22.1 days with the aeration method (Table 1). When the drip and non-aeration methods were used, high pupation rate (drip: $87.4 \%$, nonaeration: $83.6 \%$ ) and emergence rate for larvae $(82.3 \%$ and $79.4 \%)$ and pupae (94.1\% and $94.9 \%)$ were observed. On the other hand, the corresponding rates in the aeration method were low $(9.5,3.3$ and $34.2 \%$ respectively), showing high larval and pupal mortality. There was no significant difference in the sex ratio among the different rearing methods; however, more females emerged than males in each rearing method.

Effect of larval food volume: The mean number of days to pupation was 12.9 days for the standard food volume, 12.8 days for 1.5 times the standard, and 14.4 days for 0.5 times the standard (Table 1). Compared to the standard food volume, the pupation rate and emergence rate for larvae were low when both small and large food volume were provided although larvae developed rapidly with large food volume.

Effect of larval density: The effects of larval density were marked on larval development (Table 1). High larval density (400 and 500 per pan) prolonged the larval stage and lowered pupation and emergence rates. On the other hand, when larval density was 100, 200 and 300 per pan, the number of days to pupation was $12.3,13.0$ and 12.8 days, respectively, and pupation and emergence rates were higher (more than 80\%), indicating low larval and pupal mortality. The sex ratio was $1.18-$ 1.36 with density of $100-300$. The ratio at high larval density (400 and 500) was 0.82-0.87, indicating more males than females.

\section{Insemination and feeding rates}

Insemination and feeding rates from 1 st to 7 th generations in the large cage are shown in Table 2. The insemination rate of 1 st and 2 nd generations was as high as $100 \%$ and $80 \%$, respectively. Although the rate fell from the 3rd generation, after that the rate was relatively constant, 46.7$60.0 \%$. On the same day the rest of the females in the cage were fed on a mouse the whole night. Next day, the number of fed females was counted. An. minimus fed easily on the mouse in laboratory conditions. The feeding rate was $70.7-100 \%$ and high through the generations.

Table 2. Feeding and insemination rates (\%) of Anopheles minimus from Ishigaki Island, the Ryukyu Archipelago in the large cage.

\begin{tabular}{cccc}
\hline \hline Generation & No. of females examined & Insemination rate ${ }^{\mathrm{I})}$ & ${\text { Feeding rate }(\mathrm{no}, \mathrm{fed})^{\mathrm{I})}}^{\text {F1 }}$ \\
F2 & 22 & 100.0 & $100.0(7)$ \\
F3 & 40 & 80.0 & $88.0(22)$ \\
F4 & 37 & 46.7 & $95.5(21)$ \\
F5 & 97 & 53.3 & $82.9(68)$ \\
F6 & 106 & 60.0 & $97.8(89)$ \\
F7 & 189 & 53.3 & $70.7(123)$ \\
Total & 258 & 60.0 & $76.1(185)$ \\
\hline
\end{tabular}

1) Ten days after emergence, 15 females were dissected, and the rest in the cage was fed on mouse in the same day. 


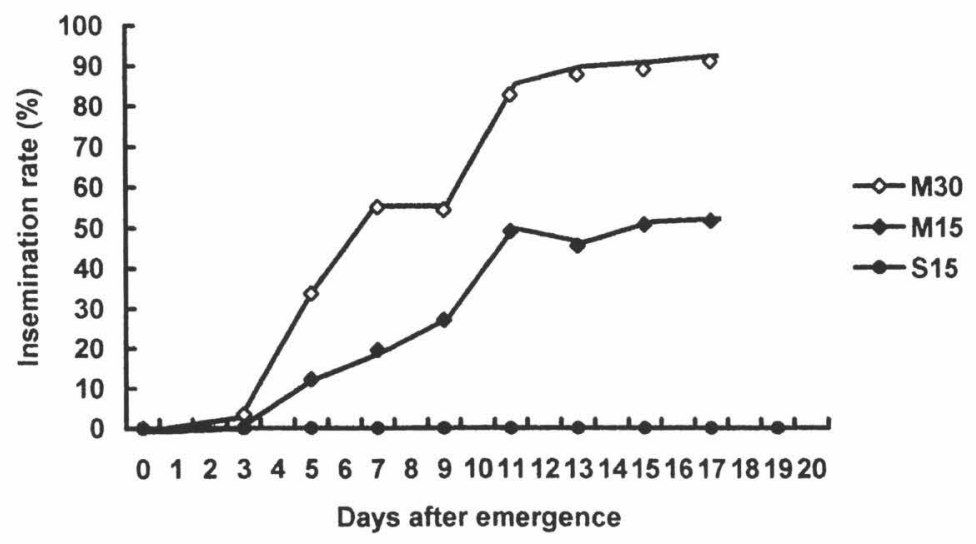

Fig. 1. Insemination rate of Anopheles minimus from Ishigaki Island, the Ryukyu Archipelago under conditions of different cage size and density. M30, 30 pairs in a medium cage; M15, 15 pairs in a medium cage; S15, 15 pairs in a small cage.

Table 3. Oviposition rate (\%) and the number of eggs of Anopheles minimus by individual rearing in small vials and group rearing in small and medium cages.

\begin{tabular}{|c|c|c|c|}
\hline \multirow{2}{*}{ Oviposition method } & \multirow{2}{*}{$\begin{array}{c}\text { Individual } \\
\text { Vial }\end{array}$} & \multicolumn{2}{|c|}{ Group } \\
\hline & & Small & Medium \\
\hline Size & $3.0(\mathrm{D}) \times 5.0(\mathrm{H}) \mathrm{cm}$ & $12 \times 12 \times 16 \mathrm{~cm}$ & $20 \times 20 \times 30 \mathrm{~cm}$ \\
\hline No. experiments conducted & 3 & 2 & 2 \\
\hline Total no. fed females (A) & 104 & $30^{4)}$ & $30^{4)}$ \\
\hline Total no. females oviposited (B) & 33 & - & - \\
\hline Oviposition rate $(\mathrm{B} / \mathrm{A})$ & 31.7 & - & - \\
\hline Total no. eggs oviposited (C) & $2,900^{1)}$ & 584 & 81 \\
\hline No. eggs/females oviposited (C/B) & $100.0^{2)}$ & - & - \\
\hline No. eggs/fed females (C/A) & $64.4^{3)}$ & 19.5 & 2.7 \\
\hline
\end{tabular}

1) Oviposited by 29 females

2) $2,900 / 29$ (4 females out of 33 were dead)

3) $2,900 / 45$ (59 females out of 104 were dead)

4) 15 females/cage

When adults were housed in a small cage, none of the females was inseminated (Fig. 1). With a medium cage, the first insemination occurred on the 5th day for 15 pairs, and on the 3rd day for 30 pairs. The rate increased faster in 30 pairs than in 15 pairs, with 50\% of females inseminated by the 7 th day for 30 pairs but by the 11 th day for 15 pairs. By the 11 th day, more than $80 \%$ insemination was observed for 30 pairs.

Number of females inseminated by a single male: On the 16th day, a single male inseminated two females on the average. The maximum inseminated by a single male was 5 .

\section{Oviposition}

The number of eggs oviposited (64.4) per fed female was higher in the individual oviposition method (Table 3 ). On the other hand, the number of eggs/fed female in the group oviposition method was 19.5 with small cages and 2.7 with medium cages. The oviposition rate was $31.7 \%$ in the individual method.

\section{Discussion}

In the present study, larval develop- 
ment of An. minimus from Ishigaki Island, the Ryukyus, was affected by the rearing methods, modified rearing water conditions and larval food volume, which Wilkinson et al. (1974) suggested although not examined in their study. The breeding sites of An. minimus in the Ryukyu Archipelago were stagnant pools of clear running streams at the forest edge but not those with high organic matters such as paddy-fields and swamps (Miyagi and Toma, 1978, 1980). The drip method newly devised in our laboratory, for rearing of Anopheles saperoi larvae which breed in edges of small streams (Toma et. al., 2001), produced water circulation, and kept the water condition clean, hence simulating a clear stream. In the present study, this method resulted in low larval mortality although it was time-consuming to prepare. The non-aeration method which was easy to use, resulted in short larval developmental period and higher pupation and emergence rate (Table 1). However pupation and emergence rate were decreased due to poor water quality under the conditions with high food volume and the non-aeration method (Table 1). It is, therefore, recommended that larval rearing should be started by the drip method with estimation of proper food volume in the early generations. After that, the non-aeration method could be utilized.

The aeration method might be appropriate to avoid scum on the water surface and to keep the water conditions clean; however, the aeration method caused very high larval mortality, especially in the earlier stage in this study. The food on the water surface was trapped in the bubbles and sank quickly to the bottom, and larvae could not obtain enough food for development. Therefore the aeration method is not suitable for larval rearing of An. minimus.

The effect of larval density on larval development also was confirmed in the present study. Larval mortality of $A n$. minimus under high-density condition was high (400 larvae/pan $=3 \mathrm{~cm}^{2} /$ larva: $39.6 \%$; 500 larvae $/$ pan $=2.4 \mathrm{~cm}^{2} /$ larva: $64.0 \%)$ as shown by Ye et al. (1982) (1.5-3 $\mathrm{cm}^{2} /$ larvae, $14.2-31.8 \%$ ), compared to those under low-density condition (100300 larvae/pan $=4-12 \mathrm{~cm}^{2} /$ larvae: $2.2-$ $14.9 \%$ ) in our experiments. Larval developmental period also was prolonged at 18.5 days in extremely high density (500 larvae/pan), while 12.3-13.0 days in the density of 100-300 larvae/pan. Since food volume per larva was constant in the present study, high larval mortality at high density might not be caused by food deficiency but intra-specific competition for limited space as in $A n$. stephensi Liston (Reisen, 1975) or deterioration of water quality due to excretion by the larvae (Ikeshoji, 1993).

Sex ratio was biased to females in the larval experiments of this study. In the study of Wilkinson et al. (1974), excess of An. minimus females from Thailand was also encountered. Ye et al. (1982) also reported biased sex ratio in $A n$. minimus from China, showing that males (52.3\%) were predominated over females (47.7\%) in their study. This may be due to selective mortality to either sex by the rearing techniques rather than to a population characteristic, since generally it would be assumed that the sex ratio in a population was $1: 1$.

Rearing at high larval density (400 and 500 larvae/pan) produced more males than females. The results indicated that high larval density caused especially high mortality of females at the larval stage and, therefore, a serious effect on the next generation. Abnormal recovery of one sex over the other under high larval density rearing was often observed in Anopheles mosquitoes such as $A n$. sacharovi Favre, (Zakharova et al., 1990) and An. stephensi (Reisen, 1975).

The insemination rate of females was influenced by space size and adult density. In this study insemination did not occur in a small sized cage. This might be because they were unable to form a swarm in the 
limited space as described for An. minimus species A (Somboon and Suwonkerd, 1997). To overcome the difficulty of mating in the laboratory, Wilkinson et al. (1974) and Ye et al. (1982) used an artificial mating technique. However, the technique is time-consuming. In the present study, we attempted colonization by natural mating, and a larger cage $(60 \times 60 \times 30$ $\mathrm{cm}$ ) was used for adaptation to laboratory conditions during the first several generations ( 1 st to 7 th), because the insemination rate in the medium cage was very low. With the larger cage, more than 50\% insemination rate was observed by 10 days after emergence (average 64.8\%, Table. 2). The higher insemination rate for the first 2 generations than the latter may be due to the fact, that a small number of females which survived were relatively healthy to mate and feed in laboratory conditions. After the 3rd generation, though the insemination rate was decreased, the rate was relatively constant at about 50\% and the strain was successfully maintained (Table 2). Ye et al. (1982) also described that success of colonization by natural mating was achieved by the insemination rate in a cage of $22 \times 22 \times 30 \mathrm{~cm}$ at the level of $54.7 \%$.

In our study, $A n$. minimus from Ishigaki Island was able to mate naturally in the space of $20 \times 20 \times 30 \mathrm{~cm}$-sized cage in a relatively earlier generation (7th generation) compared with Ye et al. (1982) with more than 24 generations in a cage of $22 \times 22 \times$ $30 \mathrm{~cm}$ and Muirhead-Thomson (1941) whose attempt failed in a $60 \times 60 \times 60 \mathrm{~cm}$ sized cage. These results might suggest difference characteristic somewhat among populations of $A n$. minimus.

The insemination rate of $A n$. minimus from Ishigaki Island, the Ryukyus, increased with the adult density as reported for An minimus sp. A (Somboon and Suwonkerd, 1997). A single male inseminated several females in this study. Higher density of males may have contributed to increasing the insemination rate. It is necessary to evaluate suitable density using the medium cage because high density may cause competition for space and high mortality.

The number of eggs oviposited was higher in the females individually housed in small vials. Small vials should be used as the oviposition method to obtain a larger number of eggs. The number of eggs decreased by the group oviposition method in the small/medium sized cages in spite of the ability to lay more than 60 eggs per female. This may be due to not incomplete blood feeding but oviposition behavior. In nature, Anopheles mosquitoes were considered to oviposit in flight, and it was observed that outdoor- caged females dropped only a few eggs at once, and then repeated the oviposition behavior frequently (Russell and Rao, 1942; Clements, 1999). In a laboratory also, Anopheles mosquitoes may perform oviposition in flight if the size of space in which they were placed was large enough. However, when a number of females were placed in a limited space in which the females could barely lay eggs in flight, the behavior was probably interfered by each other and the opportunities of oviposition by one female reduced as a result. Therefore, it might be difficult to lay all eggs and fewer eggs were obtained than expected with group rearing in the cages used in our study. On the other hand, in individual rearing with a small vial in this study, a female was almost in trapped condition in which the females had no interference with each other and oviposited in settled conditions rather than in flight. In such a situation, the female may lay more eggs than those with group rearing in the cages. These results were supported by McCrea (1984) in which females of $A n$. gambiae s. 1. that settled tended to lay all eggs. The importance of cage size may be different according to mosquito species.

The present study showed that the following conditions were suitable for rearing and obtaining a large number of $A n$. minimus originated from Ishigaki Island, the Ryukyus: (1) larvae should be reared 
by the drip method in the early generation to estimate suitable food volume and after that, by the non-aeration method; (2) 200300 larvae per pan with food volume of 25 $\mathrm{mg}$ for the $1 \mathrm{st}, 50 \mathrm{mg}$ for the $2 \mathrm{nd}, 100 \mathrm{mg}$ for the $3 \mathrm{rd}$ and $150 \mathrm{mg}$ for the 4 th-instar larvae; (3) adult cage larger than at least medium-sized cage $(20 \times 20 \times 30 \mathrm{~cm})$; and (4) individual oviposition method in small vials.

\section{ACKNOWLEDGEMENTS}

We are grateful to Ms. Yuko Tokuyama for her assistance in the present study. We also thank Dr. Yong Hoi-Sen of University of Malaya for critically reviewing the manuscript.

\section{REFERENCES}

Christopher, A. G., Gass, R. F., Munstermann, L. E. and Baimai, V. 1990. Population-genetic evidence for two species in Anopheles minimus in Thailand. Med. Vet. Entomol., 4: 25-34.

Clements, A. N. 1999. The Biology of Mosquitoes. Volume 2. Sensory reception and behaviour. 740 pp. CABI Publishing, Wallingford.

Dev, V. 1996. Anopheles minimus: its bionomics and role in the transmission of malaria in Assam, India. Bull. World. Health Org., 74: 61-66.

Gerberg, E. J., Barnard, D. R. and Ward, R. A. 1994. Manual for mosquito rearing and experimental techniques. J. Am. Mosq. Control Assoc. Bull., 5 (revised): $1-98$.

Ikeshoji, T. 1993. Mosquitoes. 246 pp. University of Tokyo Press, Tokyo.

Kanda, T., Ogawa, K., Sucharit, S., Pratchyanusorn, N., Lian, C. G. and Harinasuta, C. 1984. Cytogenetic and hybridization studies among 3 strains morphologically variated and belonging to Anopheles minimus Theobald from Japan and Thailand. Cytologia, 49: 865-881.

Komalamisra, N. 1989. Genetic variability in isozymes of Anopheles minimus group from various localities in Thailand. Jpn. J. Sanit. Zool., 40: 69-80.

McCrae, A. W. R. 1984. Oviposition by African malaria vector mosquitoes. II. Effects of site tone, water type and conspecific immatures on target selection by freshwater Anopheles gambiae Giles, sensu lato. Ann. Trop. Med. Parasitol., 78: 307-318. Miyagi, I. and Toma, T. 1978. Studies on the mosquitoes in the Yaeyama Islands 1. Appearances of Anopheline mosquitoes, especially Anopheles minimus minimus Theobald in Ishigakijima and Iriomotejima. Jpn. J. Sanit. Zool., 29: 243-250. (In Japanese with English summary)

Miyagi, I. and Toma, T. 1980. Studies on the mosquitoes in Yaeyama Islands, Japan 5. Notes on the mosquitoes collected in forest areas of Iriomotejima. Jpn. J. Sanit. Zool., 31: 81-91. (In Japanese with English summary)

Miyagi, I., Toma, T., Malenganisho, W. L. M. and Uza, M. 1996. Historical review of mosquito control as a component of malaria eradication program in the Ryukyu Archipelago. Southeast Asian J. Trop. Med. Pub. Health, 27: 498-511.

Muirhead-Thomson, R. C. 1941. Studies on the behaviour of Anopheles minimus. Part V. The behaviour of adults in relation to feeding and resting in houses. J. Malaria Inst. India, 4: 217-245.

Nutsathapana, S., Sawasdiwongphorn, P., Chitprarop, U. and Cullen, J. R. 1986a. The behaviour of Anopheles minimus Theobald (Diptera: Culicidae) subjected to differing levels of DDT selection pressure in northern Thailand. Bull. Entomol. Res., 76: 303312 .

Nutsathapana, S., Sawasdiwongphorn, P., Chitprarop, U., Cullen, J. R., Gass, R. F. and Green, C. A. 1986b. A mark-release-recapture demonstration of hostpreference heterogeneity in Anopheles minimus (Diptera: Culicidae) in a Thai village. Bull. Entomol. Res., 76: 313-320.

Reisen, W. K. 1975. Intraspecific competition in Anopheles stephensi Liston. Mosq. News, 35: 473-482.

Russell, P. F. and Rao, T. R. 1942. On the swarming, mating, and ovipositing behavior of Anopheles culicifacies. Am. J. Trop. Med., 22: 417-427.

Sawabe, K., Takagi, M., Tsuda, Y., Tang, L. H., Xu, J. J., Qui, C. P., Jin, L. Z. and Luo, X. F. 1996. Genetic differentiation among three populations of Anopheles minimus of Guangxi Yunnan Provinces in the People's Republic of China. Southeast Asian J. Trop. Med. Health, 27: 818-827.

Somboon, P. and Suwonkerd, W. 1997. Establishment of a stenogamous colony of Anopheles minimus species A. Ann. Trop. Med. Parasitol., 91: 673676.

Somboon, P., Walton, C., Sharpe, R. G., Higa, Y., Tuno, N., Tsuda, Y. and Takagi, M. 2001. Evidence for a 
new sibling species of Anopheles minimus from the Ryukyu Archipelago, Japan. J. Am. Mosq. Control Assoc., 17: 98-113.

Sucharit, S., Komalamisra, N., Leemingsawat, S., Apiwathnasorn, C. and Thongrungkiat, S. 1988. Population genetic studies on the Anopheles minimus complex in Thailand. Southeast Asian J. Trop. Med. Health, 19: 717-723.

Sucharit, S., Surathinth, K., Chaisri, U., Thongrungkiat, S. and Samang, Y. 1995. New evidence for the differed characters of Anopheles minimus species complex. Mosq. Borne Dis. Bull., 12: 1-6.

Toma, T., Higa, Y., Malenganisho, W. L. M. and Miyagi, I. 2001. Study on the biology of Anopheles saperoi (Diptera: Culicidae) for maintainance under laboratory colonization. Med. Entomol. Zool., 52: 219-226.

Tsuda, Y., Takagi, M., Toma, T., Sugiyama, A. and Miyagi, I. 1999a. Mark-release-recapture experiment with adult Anopheles minimus (Diptera: Culicidae) on Ishigaki Island, Ryukyu Archipelago, Japan. J. Med. Entomol., 36: 601-604.

Tsuda, Y., Takagi, M. and Swonkere, W. 1999b. A mark-release-recapture study on the spatial distribution of host-seeking Anophelines in Northern Thailand. J. Vecor Ecol., 25: 16-22.

Van Bortel, W., Trung, H. D., Manh, N. D., Roelants, P., Verle, P. and Coosemans, M. 1999. Identification of two species with the Anopheles minimus complex in Northern Vietnam and their behavioural divergences. Trop. Med. Int. Health, 4: 257-265.

Wilkinson, R. N., Gould, D. J. and Boonyakanist, A. 1974. Laboratory colonization of Anopheles minimus Theobald. Mosq. News, 34: 29-32.

Ye, Y. Y., Xu, Z. G., He, D. X. and Shao, B. Q. 1982. Laboratory rearing of Anopheles minimus. Acta Entomol. Sin., 25: 117-120. (In Chinese)

Yuan, Y. 1987. Studies on the two forms of Anopheles (Cellia) minimus Theobald, 1901 in China (Dip tera: Culicidae). Mosq. Syst., 19: 143-145.

Zakharova, N. F., Losev, G. I. and Iakubovich, V. I. 1990. The effect of density and temperature on the larval populations of the malaria vector Anopheles sacharovi. Med. Parazitol., 1: 3-7. (In Russian with English summary) 